\title{
Characterization of the equine skeletal muscle transcriptome identifies novel functional responses to exercise training
}

\author{
Beatrice A McGivney1, Paul A McGettigan1', John A Browne1, Alexander CO Evans13,3, Rita G Fonseca2, \\ Brendan J Loftus ${ }^{3}$, Amanda Lohan³, David E MacHugh1,3, Barbara A Murphy' , Lisa M Katz² and Emmeline W Hill*1
}

\begin{abstract}
Background: Digital gene expression profiling was used to characterize the assembly of genes expressed in equine skeletal muscle and to identify the subset of genes that were differentially expressed following a ten-month period of exercise training. The study cohort comprised seven Thoroughbred racehorses from a single training yard. Skeletal muscle biopsies were collected at rest from the gluteus medius at two time points: $T_{1}$ - untrained, ( $9 \pm 0.5$ months old) and $T_{2}$ - trained ( $20 \pm 0.7$ months old).

Results: The most abundant mRNA transcripts in the muscle transcriptome were those involved in muscle contraction, aerobic respiration and mitochondrial function. A previously unreported over-representation of genes related to RNA processing, the stress response and proteolysis was observed. Following training 92 tags were differentially expressed of which 74 were annotated. Sixteen genes showed increased expression, including the mitochondrial genes ACADVL, MRPS21 and SLC25A29 encoded by the nuclear genome. Among the 58 genes with decreased expression, MSTN, a negative regulator of muscle growth, had the greatest decrease.

Functional analysis of all expressed genes using FatiScan revealed an asymmetric distribution of 482 Gene Ontology (GO) groups and 18 KEGG pathways. Functional groups displaying highly significant $(P<0.0001)$ increased expression included mitochondrion, oxidative phosphorylation and fatty acid metabolism while functional groups with decreased expression were mainly associated with structural genes and included the sarcoplasm, laminin complex and cytoskeleton.
\end{abstract}

Conclusion: Exercise training in Thoroughbred racehorses results in coordinate changes in the gene expression of functional groups of genes related to metabolism, oxidative phosphorylation and muscle structure.

\section{Background}

The phenotypic and biochemical changes occurring in response to exercise training have been extensively studied in humans and mammals, the results mainly being of a descriptive nature. The adaptive response to training is dependent on variations in exercise-induced changes in muscle load, energy requirements and calcium flux. Endurance training results in increased aerobic capacity [1], mitochondrial biogenesis [2] and a shift from carbo-

\footnotetext{
* Correspondence: emmeline.hill@ucd.ie

${ }^{1}$ Animal Genomics Laboratory, UCD School of Agriculture, Food Science and Veterinary Medicine, College of Life Sciences, University College Dublin, Belfield, Dublin 4, Ireland

Full list of author information is available at the end of the article
}

hydrate to fat metabolism [3] whereas resistance training promotes protein synthesis $[4,5]$, muscle hypertrophy [6] and a switch from slow to fast twitch muscle. Numerous equine studies have also confirmed an increase in $\mathrm{VO}_{2 \max }$ and an increase in oxidative enzyme activity [7-12] following endurance training. An increase in type II and a concurrent decrease in type IIX fibres is observed in Thoroughbreds in response to high intensity training $[13,14]$. Also, anerobic capacity and speed and strength have been observed to increase following short duration high intensity ( 100-150\% $\left.\mathrm{VO}_{2 \max }\right)$ exercise [14-16].

In contrast, much less is known regarding the transcriptional reprogramming underlying the highly specific adaptive responses to endurance and resistance exercise. 
Exercise studies using human subjects and animal models have demonstrated that changes in the expression of a wide range of mRNA transcripts play a major role in the recovery of muscle following exercise with the expression levels of most genes returning to baseline within 24 hours [17-23]. However, it appears that repeated bouts of exercise lead to new basal levels of gene expression in resting muscle. Higher levels of mitochondrial genes and genes involved in energy metabolism were observed in endurance trained athletes compared to sedentary subjects [24]. Further evidence for a new steady state level of exercise related genes comes from a recent study in which differential levels of gene expression were observed in resting skeletal muscle from sedentary, endurance trained and resistance trained subjects. However the use of intra rather than inter-individual genetic comparisons as well as different training stimuli may have contributed to the observed differences in gene expression between the groups. The majority of differentially expressed genes were common to both trained states [25]. A surprisingly small number of genes were differentially expressed between endurance trained and resistance trained subjects given the very different phenotypic changes and distinct signalling pathways [26,27] associated with each form of exercise. Studies have indicated that concurrent endurance and resistance training results in impaired strength development and aerobic capacity when compared to a training regime with a single exercise mode [28-31] a phenomenon described as the interference effect. However, conflicting studies have found little or no effect of a combined training regime on strength and aerobic capacity [32-35]. The aim of this study was to investigate the global transcriptional response in skeletal muscle to a training regime combining endurance and high intensity sprint exercise in Thoroughbred racehorses. We hypothesise that following training differential expression of genes related to both aerobic capacity and muscle hypertrophy will be observed reflecting the dual nature of the training regime.

The Thoroughbred is a novel and valuable model for identifying molecular mechanisms basic to both endurance and resistance adaptive responses. Competitive horse racing dates to $4500 \mathrm{BC}$ and Thoroughbreds have been bred for speed and stamina since the $1700 \mathrm{~s}$. This intense selection has resulted in a highly adapted athlete [36]. Thoroughbreds have a very high aerobic capacity or maximal oxygen uptake $\left(\mathrm{VO}_{2 \max }\right)$ [37] relative to their body mass. For instance, $\mathrm{VO}_{2 \max }$ can reach 180-200 mL $\mathrm{O}_{2} / \mathrm{min} / \mathrm{kg}$, approximately 2.5 times higher than other species of similar size[38]. This is achieved through a large lung volume, high cardiac output, high haemoglobin concentration, high muscle mitochondrial volume and a high skeletal muscle mass [38-44]. During intense exercise such as under racing conditions a Thoroughbred may increase its metabolic rate from basal levels by up to 60-fold [45]. Furthermore, the Thoroughbred has a very high skeletal muscle mass comprising over $55 \%$ of total body mass [46].

A Thoroughbred racehorse trained for flat racing undergoes a training regime comprising intermittent days of sprint exercise to promote increased muscle mass among periods of prolonged exercise at a slower pace to enhance aerobic capacity. In a previous study we detected molecular signatures of both endurance and resistance exercise in untrained Thoroughbred skeletal muscle following a single bout of exhaustive exercise [47]. A further advantage of using Thoroughbreds as an exercise model is that inter-individual comparisons can be made between subjects that come from a similar background (genetic and environmental) and have undergone a similar exercise regime with relatively little variation in management. Variations in genetic and environmental conditions cannot be controlled to the same extent in human subjects.

To-date the main approach to investigating global transcriptional changes has been the use of gene expression microarray platforms [47-49]. In this study we have used digital gene expression (DGE, Illumina) profiling to characterize the assembly of genes expressed in equine skeletal muscle and to identify the subset of genes that were differentially expressed following a ten month period of exercise training. DGE is a recently developed alternative to microarray gene expression profiling [50-52]. The DGE method involves the generation of a cDNA library with a 17 bp tag generated by restriction digestions for each mRNA transcript. The tags are directly sequenced using the Illumina Genome Analyzer creating millions of short reads. In contrast to microarray technology which is limited to the hybridisation of cDNA to probes printed on the array platform, DGE is not dependent on currently available genome sequence and thus provides a global, hypothesis-free quantitative analysis of the transcriptome. The technique is conceptually similar to serial analysis of gene expression (SAGE) [53] but substantially less expensive, more general and capable of delivering more information.

Using this technique we investigated 1) the overrepresentation of functional groups in skeletal muscle relative to the entire genome, 2) the genes differentially expressed in trained relative to untrained skeletal muscle, and 3) the overrepresentation of functional groups in genes differentially expressed following training in skeletal muscle.

\section{Results and discussion}

Representation of genes by DGE tags

A limitation of genome wide gene expression analysis using DGE is that it is not possible to evaluate the expres- 
sion of genes that do not contain a NlaIII restriction site and in some cases there is ambiguity regarding the taggene matches as a single tag may match to two or more genes. 91\% $(n=22,996)$ of equine genes with Ensembl gene IDs $(n=25,180)$ have a NlaIII restriction site but $13 \%$ of these are not unique; therefore, $78 \%(n=19,271)$ of currently annotated equine genes are quantifiable using DGE.

As poor quality sequence was obtained for one of the samples just 13 samples were used for analysis. Of the 13 samples successfully sequenced a total of 183 million raw reads were generated. Of these 119 million reads passed the Illumina pipeline quality filters. These 119 million usable reads consisted of 17.6 million unique tags. $66 \%$ of the usable reads mapped to the horse genome, $30 \%$ of the usable reads mapped to the predicted Ensembl gene restriction sites and $36 \%$ to the genomic regions.

The intragenic reads may represent regulatory noncoding RNAs or novel genes. However, more likely is the explanation that these tags are a combination of segments of genes that were excluded from the current annotation (or assembly) of the equine genome; an observation which has been previously reported [54] as well as tags containing sequencing errors. We expect that as the annotation of the horse transcriptome improves that most of the genomic tags we have sequenced will be reassigned to genic tags. In particular we believe that a de novo transcriptome assembly approach (using longer sequencing reads) of the equine muscle transcriptome would enable us to more accurately allocate tags to the correct gene models. In the absence of an accurate muscle transcriptome we believe that the Ensembl horse transcriptome, which is predominantly automatically generated and infers much of the information about gene models by homology from better annotated organisms, represents the best available option for DGE tag mapping.

The reasons that reads may not match a genomic location include ambiguous reads (same sequence tag present in more than one genomic location), reads overlapping an unannotated exon boundary, sequencing errors or single nucleotide variants present in the tag. Due to the short nature of the reads used in DGE compared to other sequencing protocols it is problematic to correct for SNPs or sequencing errors by allowing mismatched bases. Other protocols (e.g. RNAseq), which generate longer tags, can overcome this limitation but they introduce new problems, the most important of which is multiple tags per transcript and a bias towards highly expressed long transcripts [55]. The intragenic reads may represent regulatory non-coding RNAs or novel genes. However, more likely is the explanation that these tags represent segments of genes that were excluded from the current annotation (or assembly) of the equine genome; an observation which has been previously reported [54].

Only the $20 \%$ of reads that unambiguously matched Ensembl genes were used for further analysis. These represented 5,068 unique genes, $\sim 25 \%$ of annotated equine genes. As some genes were represented by multiple different transcripts these were summed to calculate the total number of reads per gene. Highly expressed genes where > 50,000 tags per million (TPM) were detected made up $39 \%$ of all annotated reads. However, the majority of unique genes were expressed at low levels (i.e. 2,200 genes, < 40 TPM) and there was an inverse relationship between the level of gene expression and the number of genes expressed (Figure 1).

\section{Functional annotation of muscle transcriptome}

Using the online tool DAVID, 448 gene ontology groups and 14 KEGG pathways were observed to be significantly $(F D R=0.05)$ overrepresented in skeletal muscle relative to the entire genome. There was a substantial overlap of genes within these functional groups resulting in the overrepresentation of a large number of functionally similar gene ontology groups. Therefore only the highly significant groups are shown in Table 1. The overrepresentation of mitochondrial genes, and genes involved in muscle contraction and metabolism concurs with current SAGE data [56]. However, an overrepresentation of genes related to RNA processing, the stress response and proteolysis has not, to our knowledge, previously been reported in the muscle transcriptome. DGE is much more sensitive to the detection of low level transcripts than SAGE and consequently provides greater coverage of the muscle transcriptome. When functional analysis of only the highly expressed genes (those comprising $>0.05 \%$ of annotated muscle transcriptome) was performed, the novel overrepresented functional groups were not identified. This indicates that these are comprised of genes expressed at relatively low levels. Furthermore, functional groups involved in muscle contraction and aerobic respiration were more significantly overrepresented among the highly expressed genes (Table 1).

A list of the most abundant genes (those comprising $>0.5 \%$ of annotated muscle transcriptome) is presented in Table 2. Just 28 genes contribute to over $50 \%$ of the annotated mRNA in equine skeletal muscle and are principally involved in muscle contraction and energy metabolism. Creatine kinase muscle $(C K M)$ was the most abundantly expressed gene in equine skeletal muscle representing $6.9 \%$ of the annotated transcriptome and creatine kinase, mitochondrial 2, (CKMT2), was ranked $20^{\text {th }}$ among the most abundantly expressed genes, making up $0.8 \%$ of the transcriptome. Human studies using SAGE have indicated that $C K M$ mRNA makes up $\sim 1 \%$ of the human skeletal muscle transcriptome and CKMT2 did not feature in 

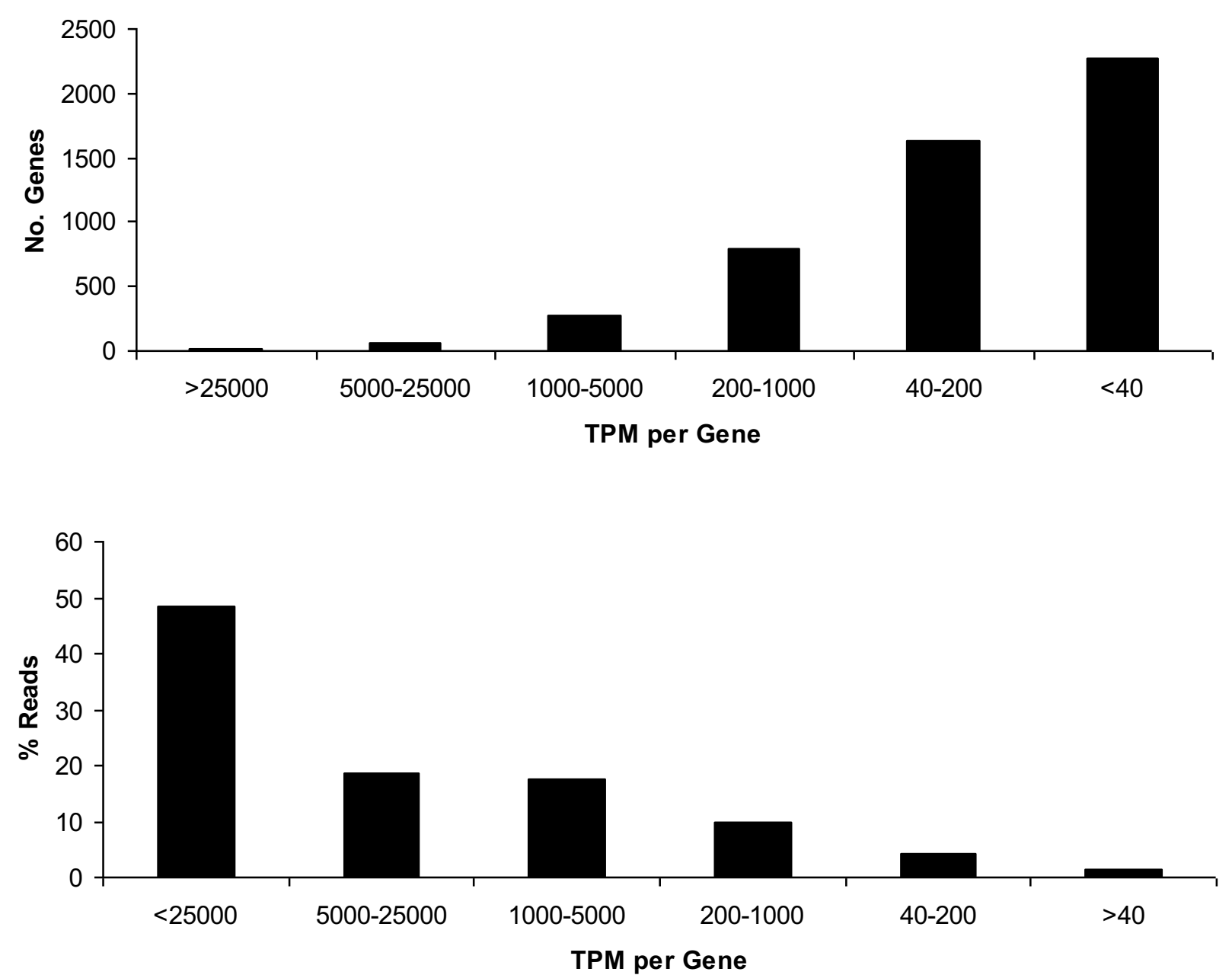

Figure 1 Relationship between the number of genes expressed and mRNA abundance. a - Number of genes compared to tags per million per gene. $b$ - The $\%$ of the total number of reads compared to the tags per million per gene.

a list of the 54 most abundantly expressed genes [56]. The very high levels of both isoforms of creatine kinase in equine muscle compared to humans is indicative of the highly adapted athletic capacity of Thoroughbred horses as creatine kinases play a crucial role as an energy store in tissues with fluctuating energy demands. CKM is utilised during anaerobic respiration while CKMT2 is tightly coupled to oxidative phosphorylation [57-60]. The importance of CKM in athletic adaptation in the horse is further supported by the identification of a novel SNP in the $C K M$ gene that, in a preliminary study, has been observed to be associated with racing performance [61]. The third most highly expressed gene in equine skeletal muscle, actin, alpha 1, skeletal muscle (ACTA1) has also been implicated as a candidate athletic performance gene following a genome scan for positive selection in Thoroughbred horses [62].

\section{Differential gene expression following training}

Following correction for multiple testing, a total of 92 transcripts were significantly $(\mathrm{FDR}=0.05)$ differentially expressed in the skeletal muscle transcriptome following a ten month period of training: nineteen transcripts showed increased expression $(+0.72$-fold to +29.3 -fold), and 73 displayed decreased expression (-0.43-fold to -4.2fold). Twenty of the differentially expressed transcripts lay within annotated genes, 54 transcripts were located < $5 \mathrm{~kb}$ up or downstream of annotated genes and for 18 transcripts no annotated genes were located within $5 \mathrm{~kb}$. The transcripts located in the vicinity of equine genes may represent regulatory regions of the genes and more in-depth analysis and annotation of the recently sequenced equine genome may lead to a reassessment of the boundaries of many currently annotated genes [63]. The uncharacterised transcripts that were not in the region of any known equine gene may represent novel 
Table 1: Functional groups of genes identified in the equine skeletal muscle transcriptome.

\begin{tabular}{|c|c|c|c|c|c|c|c|}
\hline \multirow[b]{2}{*}{ GO ID } & \multirow[b]{2}{*}{ Term } & \multicolumn{3}{|c|}{ All genes } & \multicolumn{3}{|c|}{ High abundance genes } \\
\hline & & Count & FC & Adj P & Count & FC & Adj P \\
\hline GO BP:0008152 & metabolic process & 2468 & 1.19 & $8.17 \times 10^{-55}$ & 131 & 1.2 & $4.13 \times 10^{-08}$ \\
\hline GO MF:0005515 & protein binding & 2122 & 1.35 & $4.53 \times 10^{-90}$ & 105 & 1.3 & $2.88 \times 10^{-12}$ \\
\hline GO CC:0043234 & protein complex & 713 & 1.57 & $2.75 \times 10^{-43}$ & 68 & 2.9 & $1.32 \times 10^{-15}$ \\
\hline GO CC:0005739 & mitochondrion & 407 & 1.97 & $5.63 \times 10^{-49}$ & 53 & 4.9 & $3.71 \times 10^{-23}$ \\
\hline GO BP:0006936 & muscle contraction & 73 & 1.94 & $1.78 \times 10^{-07}$ & 33 & 8.7 & $3.98 \times 10^{-17}$ \\
\hline hsa00190 & Oxidative phosphorylation & 56 & 1.55 & 0.006 & 32 & 9.5 & $4.47 \times 10^{-18}$ \\
\hline GO MF:0003723 & RNA binding & 289 & 1.76 & $3.84 \times 10^{-23}$ & 27 & 3.3 & $3.78 \times 10^{-06}$ \\
\hline GO MF:0009055 & electron carrier activity & 94 & 1.81 & $1.47 \times 10^{-07}$ & 24 & 9.4 & $1.43 \times 10^{-11}$ \\
\hline GO CC:0043292 & contractile fibre & 50 & 3.37 & $3.19 \times 10^{-17}$ & 23 & 29.9 & $5.32 \times 10^{-18}$ \\
\hline GO CC:0005840 & ribosome & 93 & 1.32 & $1.63 \times 10^{-02}$ & 21 & 5.8 & $9.29 \times 10^{-09}$ \\
\hline GO MF:0005524 & ATP binding & 434 & 1.28 & $1.71 \times 10^{-07}$ & 20 & 1.2 & NS \\
\hline GO BP:0006950 & response to stress & 337 & 1.39 & $9.17 \times 10^{-10}$ & 19 & 1.5 & NS \\
\hline GO CC:0044428 & nuclear part & 429 & 1.88 & $9.51 \times 10^{-46}$ & 10 & 0.8 & NS \\
\hline GO BP:0006457 & protein folding & 132 & 2.12 & $5.64 \times 10^{-18}$ & 10 & 3.1 & 0.007 \\
\hline GO BP:0015031 & protein transport & 290 & 1.85 & $5.91 \times 10^{-28}$ & 7 & 0.9 & NS \\
\hline GO MF:0045182 & $\begin{array}{l}\text { translation regulator } \\
\text { activity }\end{array}$ & 76 & 2.40 & $9.13 \times 10^{-13}$ & 5 & 3.2 & NS \\
\hline GO BP:0006396 & RNA processing & 195 & 1.90 & $8.21 \times 10^{-20}$ & 4 & 0.8 & NS \\
\hline GO MF:0008134 & $\begin{array}{l}\text { transcription factor } \\
\text { binding }\end{array}$ & 153 & 1.67 & $1.89 \times 10^{-09}$ & 4 & 0.9 & NS \\
\hline hsa00620 & Pyruvate metabolism & 27 & 2.28 & $3.30 \times 10^{-04}$ & 4 & 3.6 & NS \\
\hline hsa00020 & Citrate cycle (TCA cycle) & 20 & 2.36 & 0.003 & 4 & 5.1 & NS \\
\hline hsa04120 & $\begin{array}{l}\text { Ubiquitin mediated } \\
\text { proteolysis }\end{array}$ & 55 & 1.49 & 0.016 & 2 & 0.6 & NS \\
\hline hsa00071 & Fatty acid metabolism & 24 & 1.89 & 0.017 & 2 & 1.7 & NS \\
\hline GO CC:0005681 & spliceosome & 78 & 2.71 & $2.04 \times 10^{-18}$ & 1 & 0.7 & NS \\
\hline hsa03050 & Proteasome & 21 & 3.38 & $2.28 \times 10^{-07}$ & 1 & 1.7 & NS \\
\hline
\end{tabular}

GO categories and KEGG pathways overrepresented among genes expressed in equine skeletal muscle compared to all annotated equine genes. Terms in italics are more significantly overrepresented among the high abundance genes. High abundance genes are defined as those comprising $>0.05 \%$ of annotated muscle transcriptome. FC - fold change; Adj P - adjusted P value using the Benjamini and Hochberg method.

equine exercise related genes or non-protein coding regulatory mRNAs. The differentially expressed transcripts, including those located within $5 \mathrm{~kb}$ of a known gene, and the associated gene names are listed in Table 3 and Table 4.

Genes with higher post-training basal mRNA levels included those involved in the mitochondria, ubiquitination and circadian rhythm regulation, whereas genes with significantly reduced mRNA were mainly associated with cytoskeletal structure and the control of growth and development.

\section{Functional profiling of differentially expressed genes}

Significantly up and down-regulated blocks of functionally related genes were identified by performing a gene enrichment test (FatiScan) for all expressed genes ranked according to differential expression following training. Among the up-regulated genes we identified 275 significantly (FDR < 0.05) overrepresented GO terms and 13 KEGG pathways. Among the down-regulated genes we identified 207 significantly (FDR $<0.05$ ) overrepresented GO terms and five KEGG pathways. Subsets of the functional groups are shown in Table 5 and Table 6 , and were 
Table 2: Highly abundant transcripts in equine skeletal muscle

\begin{tabular}{|c|c|c|c|c|c|c|c|c|}
\hline Gene symbol & Gene name & Total reads & $\%$ All & Cumulative \% & $\begin{array}{l}\text { Average no. } \\
\text { reads pre- } \\
\text { training }\end{array}$ & $\%$ All & $\begin{array}{l}\text { Average no. } \\
\text { reads post } \\
\text { training }\end{array}$ & $\%$ All \\
\hline CKM & Creatine kinase, muscle & 236,942 & 6.9 & 6.9 & 18,642 & 6.7 & 17,870 & 7.1 \\
\hline MYL1 & $\begin{array}{l}\text { Myosin, light polypeptide 1, alkali; } \\
\text { skeletal, fast }\end{array}$ & 203,634 & 5.9 & 12.8 & 17,454 & 6.2 & 14,130 & 5.6 \\
\hline ACTA1 & Actin, alpha 1, skeletal muscle & 147,884 & 4.3 & 17.1 & 15,065 & 5.4 & 8,214 & 3.3 \\
\hline TNNC2 & Troponin C type 2 (fast) & 147,779 & 4.3 & 21.4 & 12,128 & 4.3 & 10,716 & 4.2 \\
\hline$A L D O A$ & Aldolase A, fructose-bisphosphate & 118,636 & 3.4 & 24.8 & 9,506 & 3.4 & 8,800 & 3.5 \\
\hline$\pi T N$ & Titin & 116,385 & 3.4 & 28.2 & 9,919 & 3.5 & 8,125 & 3.2 \\
\hline$M Y L P F$ & $\begin{array}{l}\text { Myosin light chain, } \\
\text { phosphorylatable, fast skeletal } \\
\text { muscle }\end{array}$ & 97,094 & 2.8 & 31.0 & 7,426 & 2.7 & 7,506 & 3.0 \\
\hline MYH1 & $\begin{array}{l}\text { Myosin, heavy polypeptide 1, } \\
\text { skeletal muscle, adult }\end{array}$ & 91,271 & 2.7 & 33.7 & 8,611 & 3.1 & 5,658 & 2.2 \\
\hline TPM2 & Tropomyosin 2 (beta) & 73,395 & 2.1 & 35.8 & 6,240 & 2.2 & 5,136 & 2.0 \\
\hline ATP2A1 & $\begin{array}{l}\text { ATPase, } \mathrm{Ca}++ \text { transporting, } \\
\text { cardiac muscle, fast twitch } 1\end{array}$ & 59,889 & 1.7 & 37.6 & 5,516 & 2.0 & 3,827 & 1.5 \\
\hline RPLP1P4 & $\begin{array}{l}\text { Ribosomal protein, large, P1 } \\
\text { pseudogene } 4\end{array}$ & 55,290 & 1.6 & 39.2 & 4,340 & 1.6 & 4,178 & 1.7 \\
\hline ENO3 & Enolase 3 (beta, muscle) & 40,827 & 1.2 & 40.4 & 3,143 & 1.1 & 3,138 & 1.2 \\
\hline LOC440359 & $\begin{array}{l}\text { Similar to cold shock domain } \\
\text { protein A short isoform }\end{array}$ & 36,681 & 1.1 & 41.4 & 2,877 & 1.0 & 2,774 & 1.1 \\
\hline ATP5O & $\begin{array}{l}\text { ATP synthase, } \mathrm{H}+\text { transporting, } \\
\text { mitochondrial } \mathrm{f} 1 \text { complex, } \mathrm{O} \\
\text { subunit }\end{array}$ & 34,234 & 1.0 & 42.4 & 2,212 & 0.8 & 2,994 & 1.2 \\
\hline MYOZ1 & MYOZENIN 1 & 32,638 & 0.9 & 43.4 & 2,449 & 0.9 & 2,564 & 1.0 \\
\hline PYGM & Phosphorylase, glycogen; muscle & 31,710 & 0.9 & 44.3 & 2,895 & 1.0 & 2,048 & 0.8 \\
\hline TPM1 & Tropomyosin 1 (alpha) & 30,823 & 0.9 & 45.2 & 2,820 & 1.0 & 1,986 & 0.8 \\
\hline MYBPC2 & $\begin{array}{l}\text { Myosin binding protein C, fast } \\
\text { type }\end{array}$ & 28,386 & 0.8 & 46.0 & 2,409 & 0.9 & 1,990 & 0.8 \\
\hline TNNT3 & Troponin t type 3 (skeletal, fast) & 27,469 & 0.8 & 46.8 & 1,960 & 0.7 & 2,244 & 0.9 \\
\hline CKMT2 & $\begin{array}{l}\text { Creatine kinase, mitochondrial } 2 \\
\text { (sarcomeric) }\end{array}$ & 27,394 & 0.8 & 47.6 & 1,622 & 0.6 & 2,523 & 1.0 \\
\hline TNNI2 & Troponin I type 2 (skeletal, fast) & 25,005 & 0.7 & 48.3 & 1,674 & 0.6 & 2,138 & 0.8 \\
\hline PGK1 & Phosphoglycerate kinase 1 & 24,378 & 0.7 & 49.0 & 2,005 & 0.7 & 1,764 & 0.7 \\
\hline RPL30 & Ribosomal protein L30 & 23,575 & 0.7 & 49.7 & 2,162 & 0.8 & 1,515 & 0.6 \\
\hline NDUFAB1 & $\begin{array}{l}\text { NADH dehydrogenase } \\
\text { (ubiquinone) 1, alpha/beta } \\
\text { subcomplex, 1, } 8 \mathrm{kDa}\end{array}$ & 22,285 & 0.6 & 50.4 & 1,570 & 0.6 & 1,838 & 0.7 \\
\hline$S L C 25 A 4$ & $\begin{array}{l}\text { Solute carrier family } 25 \\
\text { (mitochondrial carrier; adenine } \\
\text { nucleotide translocator), member } 4\end{array}$ & 21,881 & 0.6 & 51.0 & 1,270 & 0.5 & 2,037 & 0.8 \\
\hline ACTN3 & Actinin, alpha 3 & 21,097 & 0.6 & 51.6 & 2,042 & 0.7 & 1,264 & 0.5 \\
\hline PGAM2 & $\begin{array}{l}\text { Phosphoglycerate mutase } 2 \\
\text { (muscle) }\end{array}$ & 21,059 & 0.6 & 52.2 & 1,736 & 0.6 & 1,521 & 0.6 \\
\hline RPS13 & Ribosomal protein $\mathrm{S} 13$ & 19,063 & 0.6 & 52.8 & 1,610 & 0.6 & 1,343 & 0.5 \\
\hline
\end{tabular}


Table 3: Genes significantly up-regulated post-training compared to pre-training levels

\begin{tabular}{|c|c|c|c|c|}
\hline Tag & Gene symbol & Gene name & FC & Adj $P$ \\
\hline gctgctctgcagtctga & $A C A D V L$ & Acyl-Coenzyme A dehydrogenase, very long chain & 0.72 & 0.037 \\
\hline gaataattgaagactgg & ACTR3B & Arp3 Actin-Related Protein 3 Homolog B & 2.37 & 0.041 \\
\hline gcgtccttgaggtccgg & C14orf153* & Chromosome 14 open reading frame 153 & 1.14 & 0.049 \\
\hline ctgtttttctgtttttt & CUL3* & Cullin 3 & 1.43 & 0.004 \\
\hline tgataccaatattcagt & $F B X O 32^{*}$ & F-box protein 32 & 1.17 & 0.009 \\
\hline cagaaagagcagggaag & GOT1* & Glutamic-oxaloacetic transaminase 1 , soluble & 1.46 & 0.035 \\
\hline tggatgtgtggctatgg & GRHPR & Glyoxylate Reductase/Hydroxypyruvate Reductase & 1.45 & 0.043 \\
\hline ggacccatgaaggacca & IGFN1* & $\begin{array}{l}\text { Immunoglobulin-like and fibronectin type III domain-containing } \\
\text { protein } 1\end{array}$ & 2.03 & 0.019 \\
\hline tggttctgtttgttttg & KIAA1303* & P150 target of rapamycin (TOR)-scaffold protein & 2.70 & 0.049 \\
\hline gagtgcagcctttcacc & MRPS21 & $28 \mathrm{~S}$ ribosomal protein S21, mitochondrial & 3.24 & 0.024 \\
\hline accagagagatgaatgt & MRPS21 & $28 \mathrm{~S}$ ribosomal protein S21, mitochondrial & 1.76 & 0.032 \\
\hline tgttgaagcgatgcagt & PER2* & Period homolog 2 & 29.26 & 0.004 \\
\hline tgttggtaagtagatcg & PER3* & Period homolog 3 (Drosophila) & 1.05 & 0.047 \\
\hline tggctgtatggggaggc & $S L C 25 A 29^{*}$ & Solute carrier family 25 member 29 & 1.32 & 0.013 \\
\hline gccttctgcacccagaa & TNNT3* & Troponin T Type 3 (Skeletal, Fast) & 1.55 & 0.002 \\
\hline ttaaatatacttggaag & $Z A K^{*}$ & Sterile alpha motif and leucine zipper containing kinase AZK & 2.08 & 0.023 \\
\hline
\end{tabular}

chosen to include all significant $(F D R<0.05)$ KEGG pathways and all highly significantly $(\mathrm{FDR}<0.0001)$ overrepresented functional groups satisfying $\mathrm{GO}>$ level 6 and for which at least six genes were identified.

The most significantly overrepresented cellular compartment GO groups among the genes with increased abundance post-training were mitochondrion (CC GO:0005739; $\left.P<1.04 \times 10^{-41}\right)$ and related terms such as organelle inner membrane (CC GO:0019866) and mitochondrial part (CC GO:0044429). Aerobic respiration (BP GO:0009060), oxidative phosphorylation (BP GO:0006119) and the tricarboxylic acid cycle (GO BP:0006099) were among the overrepresented GO biological processes groups. The KEGG pathways included Citrate cycle (TCA cycle) (hsa00020) and Oxidative phosphorylation (hsa00190) and multiple metabolism pathways. These transcriptional data concur with biochemical and physiological studies that have demonstrated an increase in mitochondrial volume and aerobic capacity following endurance training $[1,2]$. Although there is evidence to indicate that an increase in oxidative capacity is part of the maturation process in horses [64] it has been demonstrated that exercise training, not growth, causes increases in whole muscle activity of the oxidative enzyme succinate dehydrogynase and changes in muscle fibre type composition in young Thoroughbred horses [14]. To our knowledge, this is the first time that these
GO groups have been shown to have increased expression following exercise training.

This highlights the value of using a method such as FatiScan which incorporates all experimental data rather than limiting interpretation to those that rank among the highly differentially expressed. Only three mitochondrial genes were among those significantly differentially expressed: MRPS21, SLC25A29 and ACADVL. MRPS21 is a nuclear-encoded mitochondrial ribosomal gene required for protein synthesis in the mitochondria. Therefore, an increase in mitochondrial abundance would require an increase in mitochondrial protein synthesis. The SLC25A29 and ACADVL proteins are localized in the mitochondrial inner membrane and play a role in fat metabolism $[65,66]$. The fatty acid oxidation (BP GO:0019395), fatty acid beta-oxidation (BP GO:0006635) and fatty acid metabolic process (BP GO:0006631) GO ontologies were also overrepresented among genes that increased expression following training. This is in agreement with previous observations of a shift towards fatty acid metabolism in response to exercise training [3]. Furthermore, 12 of the 13 up-regulated KEGG pathways were associated with aerobic respiration and metabolism. Overall these results demonstrate that exercise training brings about a subtle but coordinated increase in the basal level of gene expression of a wide array of genes involved in energy production and metabolism. 
Table 4: Genes significantly down-regulated post-training compared to pre-training levels

\begin{tabular}{|c|c|c|c|c|}
\hline Tag & Gene Symbol & Gene Name & FC & Adj $P$ \\
\hline acccgagagacagccga & ACTN3 & actinin, alpha 3 & -0.97 & 0.029 \\
\hline acacagttagttaattt & $A H C Y L 2^{*}$ & Putative adenosylhomocysteinase 3 & -1.39 & 0.006 \\
\hline acacagttagttaattt & $A H C Y L 2^{*}$ & Putative adenosylhomocysteinase 3 & -1.39 & 0.006 \\
\hline taattttatttttttta & ANKHD1* & Ankyrin repeat and $\mathrm{KH}$ domain containing 1 & -2.30 & 0.043 \\
\hline ttttccctcacatcttc & $A P O O L^{*}$ & Apolipoprotein O-like & -0.54 & 0.003 \\
\hline cttagtgtgtatatctc & ATP2B1 & ATPase, $\mathrm{Ca}++$ transporting, plasma membrane 1 & -0.62 & 0.041 \\
\hline atcattattttaccttt & $B C L 6^{*}$ & B-cell CLL/lymphoma 6 & -3.09 & 0.011 \\
\hline acggttttccccagatc & C1orf51* & Chromosome 1 open reading frame 51 & -1.61 & 0.035 \\
\hline cactggccaaaagattt & C21orf7 & Chromosome 21 open reading frame 7 & -3.08 & 0.035 \\
\hline ccactaccctcttactc & CALM3* & Calmodulin3 & -1.46 & 0.009 \\
\hline acagacacttggctaaa & CALM3* & Calmodulin3 & -0.83 & 0.043 \\
\hline aacagaatcaaggagct & $C C N D B P 1$ & Cyclin-D1-binding protein 1 & -0.81 & 0.035 \\
\hline gaaaacagtagctaaag & $D A G 1$ & dystroglycan 1 & -1.49 & 0.022 \\
\hline ctcaacagcaacatcaa & EIF3F & Eukaryotic translation initiation factor 3 , subunit $F$ & -0.52 & 0.002 \\
\hline tccagcctcaaagcatt & $F B X L 17^{*}$ & F-Box And Leucine-Rich Repeat Protein 17 & -0.52 & 0.002 \\
\hline aactgtagtgctttaaa & GATM & Glycine Amidinotransferase & -1.35 & 0.035 \\
\hline taggttttacctccatt & GATM $^{*}$ & Glycine Amidinotransferase & -1.35 & 0.001 \\
\hline ctggaacaggggcgaac & $G L U L^{*}$ & Glutamate-ammonia ligase & -3.94 & 0.007 \\
\hline cccatcatccccttcct & GPSN2 & GLYCOPROTEIN, SYNAPTIC 2 & -1.06 & 0.004 \\
\hline aagtcccaccccaatat & GSTM5* & Glutathione S-transferase M5 & -1.1 & 0.004 \\
\hline tccacccataagcagat & $\mathrm{HOXC9*}$ & Homeobox protein Hox-C9 & -1.04 & 0.038 \\
\hline ggactgtctttattttt & IGFBP5* & Insulin-like growth factor binding protein- 5 & -2.32 & 0.001 \\
\hline gtaaccctacacagtca & IGFBP5* & Insulin-like growth factor binding protein-5 & -2.12 & 0.038 \\
\hline cccagaaagacatttgt & $I R F 2 B P 2^{*}$ & Interferon regulatory factor 2 binding protein 2 & -1.57 & 0.001 \\
\hline caaaaggctctcctaat & KCMF1 & potassium channel modulatory factor 1 & -0.81 & 0.048 \\
\hline tttccattcaacaaaaa & KPNA1* & Karyopherin alpha 1 & -1.8 & 0.006 \\
\hline aattactctttcactgt & KPNA3* & Karyopherin alpha 3 & -1.55 & 0.043 \\
\hline cttttcacacacaaaac & LRRFIP1* & Leucine Rich Repeat & -0.95 & $4.1 \times 10^{-5}$ \\
\hline ttaagtgccattactac & МАРЗК4 & Mitogen-activated protein kinase kinase kinase 4 & -0.85 & 0.048 \\
\hline ccccaccctactcccac & $M L E C^{*}$ & Malectin & -1.13 & 0.017 \\
\hline tatgacagaaaagcaac & MSTN* & Myostatin & -2.56 & 0.002 \\
\hline atgactgtataatgtga & MSTN* & Myostatin & -2.55 & 0.008 \\
\hline gttcctaaataaataat & MSTN* & Myostatin & -4.2 & 0.004 \\
\hline ctgctgagcggectctc & MYLK2 & Myosin Light Chain Kinase 2, Skeletal Muscle & -1.94 & 0.034 \\
\hline gctcattaaagaacaaa & MYO9A* & Myosin Ixa & -1.03 & 0.043 \\
\hline ctatcttttccttttct & NAT12* & $\mathrm{N}$-acetyltransferase MAK3 homolog & -0.68 & 0.002 \\
\hline attgtttaaatatcact & NEDD4* & Neural precursor cell expressed, developmentally down-regulated 4 & -0.97 & 0.012 \\
\hline aaatcccaccctcccct & $N L N^{*}$ & Neurolysin & -1.02 & 0.008 \\
\hline tccagctttctattctt & PALLD* & Palladin, cytoskeletal associated protein & -1.06 & 0.039 \\
\hline cttctttccccacctcc & PCBP4 & Poly $(\mathrm{rC})$ binding protein 4 & -0.82 & 0.004 \\
\hline taattgcagtttactat & GNPNAT1* & Similar to glucosamine-phosphate $\mathrm{N}$-acetyltransferase 1 & -1.23 & 0.006 \\
\hline ctctagaacatttacct & PTDSS1* & Phosphatidylserine synthase 1 & -0.62 & 0.003 \\
\hline agagtcaatataaaggt & PTPLA* & Protein Tyrosine Phosphatase-Like & -2.07 & 0.01 \\
\hline aaatctaaagttaaata & $R A B 33 B^{*}$ & RAB33B, member RAS oncogene family & -1.24 & 0.04 \\
\hline
\end{tabular}


Table 4: Genes significantly down-regulated post-training compared to pre-training levels (Continued)

\begin{tabular}{|c|c|c|c|c|}
\hline cagccctgggggectac & $R Y K$ & Ryk Receptor-Like Tyrosine Kinase & -0.94 & 0.005 \\
\hline attcccatttctagtaa & SEPT7* & Transcribed locus & -0.72 & 0.018 \\
\hline tgaggacaaagctcagg & $S L C 10 A 2^{*}$ & Solute carrier family 10 & -4.11 & 0.001 \\
\hline ccttcaactcaacaaat & SNAP29* & Synaptosomal-associated protein, 29 kDa & -0.43 & 0.009 \\
\hline gtattgtaagatattaa & SNORA24* & Small nucleolar RNA SNORA24 & -0.89 & 0.037 \\
\hline aagcaagaaataaattt & $T E T 1^{*}$ & Tet oncogene 1 & -1.4 & 0.029 \\
\hline atgcagagcaccacaga & TLE1 & transducin-like enhancer of split 1 & -1.58 & $2.0 \times 10^{-5}$ \\
\hline actgacatatgtaaaga & TOR3A* & torsin family 3 , member A & -1.49 & 0.022 \\
\hline tgcctatcacctgccgg & TPD52* & Tumor protein D52 & -0.68 & 0.005 \\
\hline tcctttcagtcttcaca & TSPAN3 & Tetraspanin 3 & -0.58 & 0.04 \\
\hline ctttgtgacttccaagt & ULK2* & Unc-51-like kinase 2 & -0.72 & 0.018 \\
\hline aaaccatatttcttccc & $V C L^{*}$ & Vinculin & -1.13 & 0.005 \\
\hline cccaggcccgtccctgc & $\mathrm{ZC} 3 \mathrm{H}_{3}^{*}$ & Zinc finger CCCH-type containing 3 & -1.28 & 0.035 \\
\hline aagtctaacttccattt & ZNF704* & Zinc finger protein 704 & -0.93 & 0.013 \\
\hline ttagtttcttttcttta & ZWINT* & ZW10 interactor & -1.21 & $1.1 \times 10^{-4}$ \\
\hline
\end{tabular}

An asterisk $(*)$ indicates that the tag lay within $5 \mathrm{~kb}$ of the gene. FC - fold change; Adj P - adjusted $P$ value using the Benjamini and Hochberg method.

Interestingly there was also an up-regulation of $\mathrm{GO}$ terms involved in the immune response such as the KEGG pathway complement and coagulation cascades, complement activation and positive regulation of immune response. The up-regulation of the complement and coagulation cascades may be a response to exercise induced hemolysis. It has been suggested that exercise induced decreases in blood $\mathrm{pH}$ and increases in blood temperature may increase the osmotic fragility of erythrocytes. Previous studies have shown that an immune response is elicited in response to a single bout of exercise and that this response is attenuated in trained subjects. Furthermore, it appears that moderate exercise can enhance the immune response [67], whereas over-training in humans is detrimental to health and can leave athletes more susceptible to infection [68]. Overtraining in horses has been associated with increased levels of the alpha-1-antitrypsin protein [69] which is involved in protection of cells from inflammatory enzymes released from neutrophils [70]. This protein was also found to be increased in humans following a marathon run but returned to basal levels within a few hours [71]. Despite numerous studies documenting the immune response to a single bout of exercise [72-74], little is known regarding the molecular mechanisms governing the adaptations to the immune response brought about by exercise training. It has been suggested that exercise-induced reactive oxygen species (ROS) may play a major role in the modulation of the immune response following exercise [75]. It is also likely that exercise-induced muscle damage contributes to the inflammatory response [76]. The exercise regime undertaken by the horses in this study incorporated both endurance and sprint work which would be expected to elicit both increased ROS and intramuscular microtears.

Another interesting observation was the increased expression of ribosomal genes as elevated rates of protein synthesis and degradation have been reported following resistance exercise with an overall increase in protein mass $[4,77,78]$.

The down-regulated functional groups were mainly associated with structural genes and ion transport. It has been shown that the cellular response to mechanical stimuli, such as increased load, involves ECM signalling to the cytoskeleton at focal adhesion complexes via integrin receptors. Ion transport is central to muscular contraction. Calcium is the main regulatory and signalling molecule in muscle and ATP synthesis is dependent on phosphate transport. Although the down-regulation of these functional groups is counter-intuitive, the modulation of gene expression in these functional groups may reflect structural reorganization of myofibrils.

\section{Validation of a panel of genes by real time qRT-PCR}

Eleven genes represented by tags that were differentially expressed between untrained and trained skeletal muscle were selected for real time qRT-PCR validation. Four tags (acyl-coenzyme A dehydrogenase, very long chain $[A C A D V L]$, actinin, alpha 3 [ACTN3], dystroglycan 1 [DAG1] and $28 \mathrm{~S}$ ribosomal protein $\mathrm{S} 21$, mitochondrial [MRPS21]) were located within a known gene and seven (calmodulin 3 [CALM3], insulin-like growth factor binding protein-5 [IGFPB5], myostatin $[M S T N]$, period 
Table 5: Gene ontology categories with significantly increased expression post-training compared to pre-training levels

\begin{tabular}{|c|c|c|}
\hline GO ID & GO Term & $P$ \\
\hline BP:0009060 & aerobic respiration & $1.89 \times 10^{-16}$ \\
\hline BP:0046356 & acetyl-CoA catabolic process & $2.28 \times 10^{-16}$ \\
\hline BP:0006099 & tricarboxylic acid cycle & $5.83 \times 10^{-16}$ \\
\hline BP:0006100 & tricarboxylic acid cycle intermediate metabolic process & $5.92 \times 10^{-10}$ \\
\hline BP:0019395 & fatty acid oxidation & $4.65 \times 10^{-08}$ \\
\hline BP:0006119 & oxidative phosphorylation & $3.69 \times 10^{-06}$ \\
\hline BP:0006956 & complement activation & $4.07 \times 10^{-06}$ \\
\hline BP:0002455 & humoral immune response mediated by circulating immunoglobulin & $4.07 \times 10^{-06}$ \\
\hline BP:0002253 & activation of immune response & $5.31 \times 10^{-06}$ \\
\hline BP:0006635 & fatty acid beta-oxidation & $6.70 \times 10^{-06}$ \\
\hline BP:0002541 & activation of plasma proteins during acute inflammatory response & $8.46 \times 10^{-06}$ \\
\hline BP:0050778 & positive regulation of immune response & $8.46 \times 10^{-06}$ \\
\hline BP:0006631 & fatty acid metabolic process & $7.12 \times 10^{-05}$ \\
\hline CC:0005739 & mitochondrion & $1.04 \times 10^{-41}$ \\
\hline CC:0043231 & intracellular membrane-bound organelle & $8.17 \times 10^{-21}$ \\
\hline CC:0019866 & organelle inner membrane & $2.16 \times 10^{-19}$ \\
\hline CC:0044429 & mitochondrial part & $1.04 \times 10^{-16}$ \\
\hline CC:0044444 & cytoplasmic part & $6.14 \times 10^{-16}$ \\
\hline CC:0031967 & organelle envelope & $1.07 \times 10^{-08}$ \\
\hline CC:0031090 & organelle membrane & $2.25 \times 10^{-05}$ \\
\hline CC:0000793 & condensed chromosome & $5.40 \times 10^{-05}$ \\
\hline CC:0005840 & ribosome & $7.74 \times 10^{-05}$ \\
\hline KEGG ID & KEGG pathway & \\
\hline hsa04510 & Complement and coagulation cascades & $5.61 \times 10^{-06}$ \\
\hline has00750 & Vitamin B6 metabolism & $1.21 \times 10^{-04}$ \\
\hline hsa00790 & Folate biosynthesis & $1.35 \times 10^{-04}$ \\
\hline hsa00350 & Tyrosine metabolism & $3.93 \times 10^{-04}$ \\
\hline hsa00760 & Nicotinate and nicotinamide metabolism & $4.51 \times 10^{-04}$ \\
\hline hsa00020 & Citrate cycle (TCA cycle) & 0.002 \\
\hline hsa00190 & Oxidative phosphorylation & 0.002 \\
\hline hsa00500 & Starch and sucrose metabolism & 0.002 \\
\hline hsa00280 & Valine, leucine and isoleucine degradation & 0.003 \\
\hline hsa00380 & Tryptophan metabolism & 0.003 \\
\hline has 00720 & Reductive carboxylate cycle (CO2 fixation) & 0.007 \\
\hline hsa00860 & Porphyrin and chlorophyll metabolism & 0.019 \\
\hline hsa00252 & Alanine and aspartate metabolism & 0.032 \\
\hline
\end{tabular}


Table 6: Gene ontology categories with significantly decreased expression post-training compared to pre-training levels

\begin{tabular}{lll}
\hline GO ID & GO Term & $\boldsymbol{P}$ \\
\hline BP:0006817 & phosphate transport & $2.92 \times 10^{-20}$ \\
BP:0015698 & inorganic anion transport & $7.84 \times 10^{-18}$ \\
BP:0050679 & positive regulation of epithelial cell proliferation & $9.57 \times 10^{-05}$ \\
CC:0005856 & cytoskeleton & $4.00 \times 10^{-09}$ \\
CC:0044430 & cytoskeletal part & $5.59 \times 10^{-09}$ \\
CC:0016528 & sarcoplasm & $6.32 \times 10^{-07}$ \\
CC:0015629 & actin cytoskeleton & $2.11 \times 10^{-06}$ \\
CC:0016529 & sarcoplasmic reticulum & $2.93 \times 10^{-06}$ \\
CC:0005887 & integral to plasma membrane & $6.96 \times 10^{-06}$ \\
\hline KEGG ID & & $\boldsymbol{P}$ \\
\hline hsa04530 & KEGG pathway & $5.30 \times 10^{-04}$ \\
hsa04630 & & 0.008 \\
hsa04720 & Tight junction & 0.013 \\
hsa01430 & Jak-STAT signaling pathway & 0.032 \\
hsa04512 & Long-term potentiation & 0.046 \\
\hline
\end{tabular}

homolog 2 [PER2], period homolog 3 [PER3], solute carrier family 25 member 29 [SLC25A29] and troponin T type 3 [TNNT3]) were located within $5 \mathrm{~kb}$ of a known gene and were predicted to represent the gene. Primers were designed to span exons 1 and 2 or exons 2 and 3 of the gene of interest. This approach was taken to validate both the differential expression of genes and to assess the prediction that the differentially expressed tags that were identified within $5 \mathrm{~kb}$ of a known gene were indeed representative transcripts of that gene.

The mean expression of three of the four genes represented by intergenic tags reached significance $(P<0.05)$ and concurred with DGE data. ACTN3 showed the same direction of change as the DGE data and tended towards significance $(P<0.1)$. The mean expression of six of the seven genes predicted to be represented by adjacent tags agreed with the DGE data, the exception being TNNT3. The putative TNNT3 tag was matched to a region $\sim 880$ bp downstream of the TNNT3 gene and may represent a novel gene or mRNA. Alternatively the tag may span a splice site in an alternative gene and consequently may represent RNA transcribed from a different region in the genome. Real time qRT-PCR results are detailed in Table 7.

The PER2 and PER3 genes, key molecular clock components within the mammalian circadian timing system [79], had mean post-training increases in expression of +1.88 -fold and +1.74 fold respectively. The induction of these genes may represent an entrainment of the muscle transcriptional clock by a regular exercise regime. While primarily regulated by photoperiodic signals to the master pacemaker within the suprachiasmatic nucleus, peripheral circadian clocks, which are known to exist in almost all peripheral tissues examined to date [80], can also be entrained by alternative timing cues including exercise [81] and feeding [82]. The role of peripheral clocks is to align specific tissue function to the correct time of day via differential regulation of subsets of clockcontrolled genes.

As exercise is a known synchroniser of circadian rhythms in mice [83], humans [81] and horses [84], and PER2 has previously been shown to oscillate in equine tissues [85], the increased expression of PER genes posttraining in the current study is thought to represent a strengthening of the endogenous circadian clock in equine muscle. Furthermore, human studies have shown time of day variations in exercise performance at the physiological level [86-88], and it has been suggested that circadian rhythms may play an important role in sports performance [89]. Combined with our results, this is strong incentive for further investigation of the influence of training times on daily muscle function in the horse, such that optimal athletic performance may be achieved.

The proteins encoded by $A C A D V L(+1.72$-fold, $P=$ 0.014), MRPS21 (+6.03-fold, $P=0.013)$ and SLC25A29 $(+1.22$-fold, $P=0.350)$ function in the mitochondria to increase protein synthesis and fat metabolism. The increase in expression of the gene encoding the mito- 
Table 7: Real time qRT-PCR results for genes used to validate DGE data

\begin{tabular}{|c|c|c|c|c|c|}
\hline Tag & Gene Symbol & Gene Name & DGE FC & RT-PCR FC & $\mathbf{P}$ \\
\hline gctgctctgcagtctga & $A C A D V L$ & Acyl-Coenzyme A dehydrogenase, very long chain & 1.65 & 1.72 & 0.014 \\
\hline acccgagagacagccga & ACTN3 & Actinin, alpha 3 & -1.97 & -1.41 & NS \\
\hline ccactaccctcttactc & CALM3 & Calmodulin 3 & -2.75 & -1.81 & 0.028 \\
\hline gaaaacagtagctaaag & $D A G 1$ & Dystroglycan 1 & -2.81 & -1.27 & 0.021 \\
\hline ggactgtctttattttt & IGFBP5 & Insulin-like growth factor binding protein-5 & -4.35 & -3.18 & 0.023 \\
\hline gagtgcagcctttcacc & MRPS21 & $28 \mathrm{~S}$ ribosomal protein S21, mitochondrial & 9.47 & 6.03 & 0.013 \\
\hline tatgacagaaaagcaac & MSTN & Myostatin & -4.35 & -4.97 & 0.004 \\
\hline tgttgaagcgatgcagt & PER2 & Period homolog 2 & $+\inf$ & 1.88 & 0.003 \\
\hline tgttggtaagtagatcg & PER3 & Period homolog 3 (Drosophila) & 2.07 & 1.74 & 0.001 \\
\hline tggctgtatggggaggc & $S L C 25 A 29$ & Solute carrier family 25 member 29 & 2.50 & 1.22 & 0.035 \\
\hline gatgaagctgggatgca & TNNT3 & Troponin T Type 3 & 2.93 & 0.94 & NS \\
\hline
\end{tabular}

chondrial ribosomal protein MRPS21 likely reflects an increase in mitochondrial protein synthesis and an overall increase in mitochondrial volume. Numerous studies have demonstrated an increase in mitochondrial volume concurrent with an increase in $\mathrm{VO}_{2 \max }$ following endurance training [90-93]. The proteins encoded by $A C A D V L$ and SLC25A29 are involved in fat metabolism and are located in the mitochondrial inner membrane.

$A C T N 3, C A L M 3$ and DAG1 were decreased in expression by -1.41 -fold $(P=0.090),-1.81$-fold $(\mathrm{P}=0.028)$ and 1.27 -fold $(P=0.021)$ respectively. The ACTN3 protein is localized to the skeletal muscle z-discs and DAG1 forms part of the dystroglycan complex. A null mutation in the ACTN3 gene has been associated with sprint performance in human athletes [94] and DAG1 has been proposed as a candidate gene in some muscular myopathies $[95,96]$. CALM3 is an isoform of calmodulin, a calciummodulated protein which regulate numerous protein targets. The binding of calcium to calmodulin induces a conformational change which affects its ability to bind target proteins. In this manner calmodulin may be used by other proteins as a calcium sensor and signal transducer. CALM3 may be involved in muscle fibre type transformation in response to muscle excitation $[97,98]$. CALM3 gene expression was also decreased in equine muscle four hours post exhaustive treadmill exercise [47].

IGFBP5 and MSTN encode growth factors with large observed decreases in expression post training (-3.18fold, $P=0.023$ and -4.97 -fold $P=0.004$ respectively). IGFBP5 is one of family of modulators of insulin like growth factors (IGFs) which interact with IGFs resulting in an increase in half life and alteration of the interaction with receptors. IGF-1 promotes muscle hypertrophy and protein levels are increased in humans following adminis- tration of human growth hormone as an illegal doping agent $[99,100]$. The exact mode of action of IGFBP5 is poorly understood however it has been shown to associate with the extra cellular matrix and is a regulator of a wide range of physiological processes including cell proliferation and muscle cell differentiation [101-103].

Myostatin encoded by the MSTN gene is a negative regulator of muscle growth and an inhibitor of satellite cell proliferation[104]. The expression of MSTN was found to be decreased in humans following resistance training $[105,106]$. Null mutations in this gene have been found to cause a double muscling phenotype in cattle, dogs, and humans [107-111]. Structural variation in the MSTN gene has also been associated with athletic performance in dogs [110] and horses [112]. The differential expression of this gene is of particular significance as an intronic SNP in equine MSTN has been found to be a strong predictor of optimal racing distance in Thoroughbred racehorses [112].

\section{Conclusion}

Deep sequencing of the equine skeletal muscle transcriptome has revealed novel transcripts and functional groups associated with this tissue. Furthermore, following exercise training we have observed an increase in the occurrence of genes involved in metabolism and oxidative phosphorylation, and a decrease in the expression of structural genes. Overrepresented functional groups of genes post-training were associated with both endurance and resistance exercise. This study documents the transcriptome-wide reprogramming of skeletal muscle in Thoroughbred racehorses that brings about the well documented phenotypic adaptations to exercise. 


\section{Methods Subjects}

All animal procedures were approved by the University College Dublin, Animal Research Ethics Committee, a licence was granted from the Department of Health and Children (Ireland) and owners' consent was obtained for all horses.

Seven two-year-old untrained Thoroughbred horses ( $n$ $=5$ females, $n=2$ entire males), raised on the same farm for the previous 2 - 3 months and destined for Flat racing with the same trainer comprised the study cohort. The horses had a mean height of $154.9 \mathrm{~cm}( \pm 2.8)$ and a mean pre-training weight of $437.4 \mathrm{~kg}( \pm 18.0)$. All horses undertook a regular exercise regime with the same trainer for 10 months (trained). This consisted of light canter $(1,500$ $\mathrm{m}$ ) once a day six times a week on an all-weather gallop and higher intensity exercise ("work") no more than once a week which consisted of warm-up (walk and trot) followed by gallop with velocities reaching maximal intensity for $800-1,000 \mathrm{~m}$.

\section{Muscle biopsy sampling}

Percutaneous needle muscle biopsies [113] were obtained from the dorsal compartment of the gluteus medius muscle according to Dingboom and colleagues [114] using a 6 mm diameter, modified Bergstrom biopsy needle (Jørgen KRUUSE, Veterinary Supplies). Biopsies were taken approximately $15 \mathrm{~cm}$ caudodorsal (one-third of the distance) to the tuber coxae on an imaginary line drawn from the tuber coxae to the head of the tail. The biopsies were obtained at a depth of $80 \mathrm{~mm}$. Each biopsy site was shaved, scrubbed with an antiseptic and desensitized by a local anaesthetic. The biopsy samples were washed with sterile PBS (BD Biosciences, San Jose, CA) and preserved in RNAlater (Ambion, UK) for 24 hours at $4^{\circ} \mathrm{C}$ and then stored at $-20^{\circ} \mathrm{C}$. Muscle biopsy samples were collected at rest at two time points: $\mathrm{T}_{0}$-untrained and $\mathrm{T}_{2}$-trained.

\section{RNA isolation and purification}

Approximately $100 \mathrm{mg}$ of each muscle biopsy sample was removed from RNAlater and homogenized in $1 \mathrm{ml}$ TRIzol using a TissueLyser (Qiagen Ltd, Crawley, UK) and extracted according to the manufacturer's instructions. Each sample was purified using the RNeasy' Mini kit (Qiagen Ltd, Crawley, UK) and DNase treated with RNase free DNase (Qiagen Ltd, Crawley, UK). RNA was quantified using a NanoDrop ND1000 spectrophotometer V 3.5.2 (NanoDrop Technologies, Wilmington, DE) and RNA quality was subsequently assessed using the $18 \mathrm{~S} / 28 \mathrm{~S}$ ratio and RNA integrity number (RIN) on an Agilent Bioanalyser with the RNA 6000 Nano LabChip kit (Agilent Technologies Ireland Ltd, Dublin, Ireland) according to the manufacturers' instructions.

\section{Library preparation for Illumina sequencing}

The Illumina cDNA library was prepared according to the manufacturer's instructions. All reagents were supplied by Illumina apart from SuperScript II Reverse Transcriptase (part \# 18064-014) with 100 mM DTT. Briefly, $1.5 \mu \mathrm{g}$ mRNA was isolated from total RNA by binding the mRNA to a magnetic oligo(dT) bead. Double stranded cDNA was synthesized and cleaved at each NlaIII site. The site of NlaIII cleavage was ligated with an Illuminasupplied adaptor using T4 DNA ligase. The bead bound double stranded cDNA was the cut by the restriction enzyme, MmeI. This resulted in a $17 \mathrm{bp}$ tag which was no longer attached to the oligo(dT) bead. The cDNA construct was then precipitated and the site of MmeI cleavage was ligated with an Illumina-supplied adaptor using T4 DNA ligase. The adaptor ligated cDNA was PCR amplified with two adapter primers (Illumina). The PCR product of $85 \mathrm{bp}$ was purified by gel extraction in preparation for loading on the Illumina Cluster Station. The quality and quantity of the purified constructs were assessed using an Agilent DNA series 7500 series II assay (Agilent Technologies Ireland Ltd, Dublin, Ireland) and Qubit fluorometer according to manufacturer's instructions. Cluster generation and sequencing analysis were carried out using Illumina's Solexa Sequencer according to the manufacturer's instructions.

\section{Analysis}

The DGE samples were processed through the standard software pipeline provided by Illumina for the Genome Analyzer. The sequence reads were base called using the Bustard base caller (part of the Illumina software). The tag annotation pipeline consisted of two parts: mapping to known Ensembl [115] cDNAs and mapping to the genome. The known cDNAs from version 49 of Ensembl for the EquCab2 assembly of the equine genome were downloaded in FASTA format using the Ensembl biomart tool. The FASTA files for the individual equine chromosomes were downloaded from the UCSC genome browser website [116]. A pipeline consisting of perl, C++ and linux shell scripts was used to conduct an in-silico digestion of both the transcriptome and genome and to generate tag location records which were loaded into a MySQL database. The tag records were then annotated according to their type (genomic or cDNA, canonical, noncanonical, repeat etc.). A matrix of tag counts for each sample was generated. The edgeR Bioconductor package [117] was used to determine differential expression of tags in each group.

\section{Functional clustering according to gene ontology annotations}

The equine Ensembl gene IDs were cross-matched to human Ensembl gene IDs. Using the Ensembl IDs of 
Table 8: Real time qRT-PCR primers for genes used to validate DGE data

\begin{tabular}{lll}
\hline Gene symbol & Forward Primer & Reverse Primer \\
\hline ACADVL & ctgcccagcgatcctatg & ttccactggtcgaagtctca \\
ACTN3 & cggcgagtatatggaacagg & gtgagttgcaccaggcagt \\
CALM & agcacttggtggactccttg & aaatgcctgactgtgctcaa \\
DAG1 & ccaggaggagtgagcacct & ctcaccctctgcacacctg \\
IGFBP5 & ggaggagccgagaacactg & gcgaagcctccatgtgtc \\
MRPS21 & ggagatctgctgtttgctca & tctctcaaagcgacccatct \\
MSTN & tgacagcagtgatggctctt & ttgggttttccttccacttg \\
$P E R 2$ & agcctgatgatggcgaagtctgaa & agttctttgtgcgtgtctgccttg \\
$P E R 3$ & aactatgcccttcgctgtgt & gtacccggtcacatctgctt \\
SLC25A29 & ggacacccgtttgacactg & ctgatgatggattggaagca \\
$T N N T 3$ & cggagggggagaaagtagac & caaagtggctgtcgatgaga \\
\hline
\end{tabular}

human homologues of equine genes it was possible to use the Database for Annotation, Visualization and Integrated Discovery (DAVID) $[118,119]$ for functional clustering and overrepresentation analyses. The Expression Analysis Systematic Explorer (EASE) tool [120] within DAVID was used to investigate the representation of functional groups in equine skeletal muscle relative to the whole genome. The FatiScan $[121,122]$ gene enrichment test was used to analyse the transcriptional profile posttraining. FatiScan is part of the Babelomics Suite of web tools and tests for the asymmetrical distribution of biological labels in an ordered list of genes through application of a Fisher's exact test. Genes were ranked by differential expression and FatiScan was used to detect functional blocks (GO and KEGG pathways) that were significantly up-regulated and down-regulated posttraining. Results from both EASE and FatiScan were corrected for multiple testing using the Benjamini and Hochberg method [123].

\section{Real time quantitative RT-PCR}

Selected cDNA samples were quantified by real time quantitative RT-PCR (qRT-PCR). $1 \mu \mathrm{g}$ of total RNA from each sample was reverse transcribed into cDNA with oligo-dT primers using a SuperScript ${ }^{\mathrm{TM}}$ III first strand synthesis SuperMix kit according to the manufacturer's instructions (Invitrogen Ltd, Paisley, UK). The converted cDNA was diluted to $2.5 \mathrm{ng} / \mu \mathrm{l}$ working stocks and stored at $-20^{\circ} \mathrm{C}$ for subsequent analyses. Oligonucleotide primers for real time qRT-PCR were designed using Primer3 version 3.0 http://www.primer3.sourceforge.net and commercially synthesized (MWG Biotech, Germany). Primer details are shown in Table 8 . Each reaction was carried out in a total volume of $20 \mu \mathrm{l}$ with $5 \mu \mathrm{l}$ of cDNA (1 ng/ $\mu \mathrm{l}$ ), $10 \mu \mathrm{l} \mathrm{SYBR}{ }^{\circ}$ Green PCR Master Mix (Applied Biosystems, Cambridgeshire, UK) and $5 \mu$ primer $/ \mathrm{H}_{2} \mathrm{O}$. Real time qRT-PCR was performed using a 7500 Fast RealTime PCR machine (Applied Biosystems, Cambridgeshire, UK). All reactions were performed in duplicate. Hypoxanthine phosphoribosyltransferase 1 (HPRT) was selected as a stable reference gene based on a study of equine reference genes for real time qRT-PCR [124] and on the DGE results. Expression values were calculated using a standard curve which was plotted based on the expression of HPRT in serial dilutions of equine skeletal muscle RNA $(1: 1,1: 2,1: 4,1: 8,1: 16,1: 32$, and 1:64). The standard curve method was used to normalise the gene expression data. The paired Student's t-test was used to identify significant differences in mRNA abundance between time-points.

\section{Authors' contributions}

BMcG \& EH designed the experiment. BMcG, EH, LK and RF participated in the collection of samples. BMcG performed the RNA extractions. BMcG and JB generated the CDNA libraries. BMcG performed the real time aRT-PCR experiments, $\mathrm{PM}$ was responsible for the bioinformatics pipeline. $\mathrm{AL}, \mathrm{BL}$ and $\mathrm{AE}$ were responsible for the sequencing project. BMCG performed functional analysis of the data. PM, BM and DMacH assisted with manuscript preparation. BMcG and EH wrote the paper. EH coordinated and supervised the project. All authors read and approved the final manuscript.

\section{Acknowledgements}

The authors thank JS Bolger for access to horses. We thank P O'Donovan and Glebe House yard staff for assistance with sample collection. This work was financed by a Science Foundation Ireland President of Ireland Young Researcher Award (04/YI1/B539) to EH. AE and PM were funded by Science Foundation Ireland (07/SRC/B1156)

\section{Author Details}

'Animal Genomics Laboratory, UCD School of Agriculture, Food Science and Veterinary Medicine, College of Life Sciences, University College Dublin, Belfield, Dublin 4, Ireland, 2University Veterinary Hospital, UCD School of Agriculture, Food Science and Veterinary Medicine, College of Life Sciences, University College Dublin, Belfield, Dublin 4, Ireland and 3UCD Conway Institute of Biomolecular and Biomedical Research, University College Dublin, Belfield, Dublin 4, Ireland

Received: 18 February 2010 Accepted: 23 June 2010

Published: 23 June 2010 


\section{References}

1. Saltin B, Hartley LH, Kilbom A, Astrand I: Physical training in sedentary middle-aged and older men. II. Oxygen uptake, heart rate, and blood lactate concentration at submaximal and maximal exercise. Scand J Clin Lab Invest 1969, 24(4):323-334.

2. Adhihetty PJ, Irrcher I, Joseph AM, Ljubicic V, Hood DA: Plasticity of skeletal muscle mitochondria in response to contractile activity. Exp Physio/ 2003, 88(1):99-107.

3. Holloszy JO, Rennie MJ, Hickson RC, Conlee RK, Hagberg JM: Physiological consequences of the biochemical adaptations to endurance exercise. Ann N Y Acad Sci 1977, 301:440-450.

4. Hartman JW, Moore DR, Phillips SM: Resistance training reduces wholebody protein turnover and improves net protein retention in untrained young males. Appl Physiol Nutr Metab 2006, 31(5):557-564.

5. Yarasheski KE, Zachwieja JJ, Bier DM: Acute effects of resistance exercise on muscle protein synthesis rate in young and elderly men and women. Am J Physiol 1993, 265(2 Pt 1):E210-214

6. Nader GA, Esser KA: Intracellular signaling specificity in skeletal muscle in response to different modes of exercise. J Appl Physiol 2001, 90(5):1936-1942.

7. Roneus M, Essen-Gustavsson B, Lindholm A, Persson SG: Skeletal muscle characteristics in young trained and untrained standardbred trotters. Equine Vet J 1992, 24(4):292-294.

8. Roneus M: Muscle characteristics in standardbreds of different ages and sexes. Equine Vet J 1993, 25(2):143-146.

9. Katz LM, Bayly WM, Hines MT, Sides RH: Differences in the ventilatory responses of horses and ponies to exercise of varying intensities. Equine Vet J Supp/ 1999, 30:49-51

10. Serrano AL, Quiroz-Rothe E, Rivero JL: Early and long-term changes of equine skeletal muscle in response to endurance training and detraining. Pflugers Arch 2000, 441(2-3):263-274

11. Hinchcliff KW, Lauderdale MA, Dutson J, Geor RJ, Lacombe VA, Taylor LE: High intensity exercise conditioning increases accumulated oxygen deficit of horses. Equine Vet J 2002, 34(1):9-16.

12. McGowan CM, Golland LC, Evans DL, Hodgson DR, Rose RJ: Effects of prolonged training, overtraining and detraining on skeletal muscle metabolites and enzymes. Equine Vet J Supp/ 2002:257-263.

13. Rivero JL, Ruz A, Marti-Korfft S, Lindner A: Contribution of exercise intensity and duration to training-linked myosin transitions in thoroughbreds. Equine Vet J Supp/ 2006:311-315.

14. Yamano S, Eto D, Sugiura T, Kai M, Hiraga A, Tokuriki M, Miyata H: Effect of growth and training on muscle adaptation in Thoroughbred horses. Am J Vet Res 2002, 63(10):1408-1412.

15. Eto D, Yamano S, Mukai K, Sugiura T, Nasu T, Tokuriki M, Miyata H: Effect of high intensity training on anaerobic capacity of middle gluteal muscle in Thoroughbred horses. Res Vet Sci 2004, 76(2):139-144.

16. Rivero JL, Sporleder HP, Quiroz-Rothe E, Vervuert I, Coenen M, Harmeyer J: Oral L-carnitine combined with training promotes changes in skeletal muscle. Equine Vet J Supp/ 2002(34):269-274

17. Bickel CS, Slade J, Mahoney E, Haddad F, Dudley GA, Adams GR: Time course of molecular responses of human skeletal muscle to acute bouts of resistance exercise. J Appl Physiol 2005, 98(2):482-488.

18. Neufer PD, Dohm GL: Exercise induces a transient increase in transcription of the GLUT-4 gene in skeletal muscle. Am J Physiol 1993, 265(6 Pt 1):C1597-1603.

19. Yang Y, Creer A, Jemiolo B, Trappe S: Time course of myogenic and metabolic gene expression in response to acute exercise in human skeletal muscle. J Appl Physio/ 2005, 98(5):1745-1752.

20. Pilegaard H, Saltin B, Neufer PD: Exercise induces transient transcriptional activation of the PGC-1alpha gene in human skeletal muscle. J Physio/ 2003, 546(Pt 3):851-858.

21. Mahoney DJ, Parise G, Melov S, Safdar A, Tarnopolsky MA: Analysis of global mRNA expression in human skeletal muscle during recovery from endurance exercise. FASEB J 2005, 19(11):1498-1500.

22. Schmutz S, Dapp C, Wittwer M, Vogt M, Hoppeler H, Fluck M: Endurance training modulates the muscular transcriptome response to acute exercise. Pflugers Arch 2006, 451(5):678-687.

23. Klossner S, Dapp C, Schmutz S, Vogt M, Hoppeler H, Fluck M: Muscle transcriptome adaptations with mild eccentric ergometer exercise. Pflugers Arch 2007, 455(3):555-562
24. Puntschart A, Claassen H, Jostarndt K, Hoppeler H, Billeter R: mRNAs of enzymes involved in energy metabolism and mtDNA are increased in endurance-trained athletes. Am J Physiol 1995, 269(3 Pt 1):C619-625.

25. Stepto NK, Coffey VG, Carey AL, Ponnampalam AP, Canny BJ, Powell D, Hawley JA: Global gene expression in skeletal muscle from well-trained strength and endurance athletes. Med Sci Sports Exerc 2009, 41(3):546-565

26. Atherton PJ, Babraj J, Smith K, Singh J, Rennie MJ, Wackerhage H: Selective activation of AMPK-PGC-1 alpha or PKB-TSC2-mTOR signaling can explain specific adaptive responses to endurance or resistance training-like electrical muscle stimulation. FASEB J 2005, 19(7):786-788

27. Coffey VG, Zhong Z, Shield A, Canny BJ, Chibalin AV, Zierath JR, Hawley JA: Early signaling responses to divergent exercise stimuli in skeletal muscle from well-trained humans. FASEB J 2006, 20(1):190-192.

28. Coffey VG, Pilegaard H, Garnham AP, O'Brien BJ, Hawley JA: Consecutive bouts of diverse contractile activity alter acute responses in human skeletal muscle. J App/ Physio/ 2009, 106(4):1187-1197.

29. Coffey VG, Reeder DW, Lancaster Gl, Yeo WK, Febbraio MA, Yaspelkis BB, Hawley JA: Effect of high-frequency resistance exercise on adaptive responses in skeletal muscle. Med Sci Sports Exerc 2007, 39(12):2135-2144

30. Nader GA: Concurrent strength and endurance training: from molecules to man. Med Sci Sports Exerc 2006, 38(11):1965-1970

31. Hickson RC: Interference of strength development by simultaneously training for strength and endurance. Eur J Appl Physiol Occup Physiol 1980, 45(2-3):255-263.

32. Sale DG, Jacobs I, MacDougall JD, Garner S: Comparison of two regimens of concurrent strength and endurance training. Med Sci Sports Exerc 1990, 22(3):348-356

33. McCarthy JP, Agre JC, Graf BK, Pozniak MA, Vailas AC: Compatibility of adaptive responses with combining strength and endurance training. Med Sci Sports Exerc 1995, 27(3):429-436.

34. Shaw BS, Shaw I: Compatibility of concurrent aerobic and resistance training on maximal aerobic capacity in sedentary males. Cardiovas $\mathrm{J}$ Afr 2009, 20(2):104-106.

35. Davis WJ, Wood DT, Andrews RG, Elkind LM, Davis WB: Concurrent training enhances athletes' strength, muscle endurance, and other measures. J Strength Cond Res 2008, 22(5):1487-1502.

36. Poole D: Current concepts of oxygen transport during exercise. Equine and Comparative Exercise Physiology 2003, 1:5-22.

37. Young LE, Marlin DJ, Deaton C, Brown-Feltner H, Roberts CA, Wood JL: Heart size estimated by echocardiography correlates with maximal oxygen uptake. Equine Vet J Supp/ 2002:467-471.

38. Jones JH, Longworth KE, Lindholm A, Conley KE, Karas RH, Kayar SR, Taylor $C R$ : Oxygen transport during exercise in large mammals. I. Adaptive variation in oxygen demand. J App/ Physiol 1989, 67(2):862-870.

39. Banister EW, Purvis AD: Exercise electrocardiography in the horse by radiotelemetry. J Am Vet Med Assoc 1968, 152(7):1004-1008.

40. Marsland WP: Heart rate response to submaximal exercise in the Standardbred horse. J Appl Physiol 1968, 24(1):98-101.

41. Asheim A, Knudsen O, Lindholm A, Rulcker C, Saltin B: Heart rates and blood lactate concentrations of standardbred horses during training and racing. J Am Vet Med Assoc 1970, 157(3):304-312.

42. Hall MC, Steel JD, Stewart GA: Cardiac monitoring during exercise tests in the horse. 2. Heart rate responses to exercise. Australian veterinary journal 1976, 52(1):1-5.

43. Kayar SR, Hoppeler H, Lindstedt SL, Claassen H, Jones JH, EssenGustavsson B, Taylor CR: Total muscle mitochondrial volume in relation to aerobic capacity of horses and steers. Pflugers Arch 1989, 413(4):343-347.

44. Hargreaves BJ, Kronfeld DS, Naylor JR: Ambient temperature and relative humidity influenced packed cell volume, total plasma protein and other variables in horses during an incremental submaximal field exercise test. Equine Vet J 1999, 31(4):314-318

45. Guthrie AJ, Lund RJ: Thermoregulation. Vet Clin North Am, Equine Pract 1998, 14:45-59.

46. Gunn HM: Muscle, bone and fat proportions and muscle distribution of Thoroughbreds and other horses. JR Gillespie and NE Robinson, Editors, Equine exercise physiology 2 Davis, ICEEP, CA 1987:253-264.

47. McGivney BA, Eivers SS, MacHugh DE, MacLeod JN, O'Gorman GM, Park SD, Katz LM, Hill EW: Transcriptional adaptations following exercise in 
thoroughbred horse skeletal muscle highlights molecular mechanisms that lead to muscle hypertrophy. BMC Genomics 2009, 10:638.

48. Mienaltowski MJ, Huang L, Frisbie DD, Mcllwraith CW, Stromberg AJ, Bathke AC, Macleod JN: Transcriptional profiling differences for articular cartilage and repair tissue in equine joint surface lesions. BMC Med Genomics 2009, 2:60.

49. Bright LA, Burgess SC, Chowdhary B, Swiderski CE, McCarthy FM: Structural and functional-annotation of an equine whole genome oligoarray. BMC Bioinformatics 2009, 10(Suppl 11):S8.

50. Morrissy S, Zhao Y, Delaney A, Asano J, Dhalla N, Li I, McDonald H, Pandoh P, Prabhu AL, Tam A, et al.: Digital gene expression by tag sequencing on the illumina genome analyzer. Curr Protoc Hum Genet Chapter 11(Unit $1111): 11-36$

51. Serteyn $D$, Piquemal $D$, Vanderheyden L, Lejeune JP, Verwilghen $D$, Sandersen C: Gene expression profiling from leukocytes of horses affected by osteochondrosis. J Orthop Res 28(7):965-70.

52. Asmann YW, Klee EW, Thompson EA, Perez EA, Middha S, Oberg AL, Therneau TM, Smith DI, Poland GA, Wieben ED, et al.: 3 ' tag digital gene expression profiling of human brain and universal reference RNA using Illumina Genome Analyzer. BMC Genomics 2009, 10:531.

53. Velculescu VE, Zhang L, Vogelstein B, Kinzler KW: Serial analysis of gene expression. Science 1995, 270(5235):484-487.

54. Coleman SJ, Zeng Z, Mienaltowske M, Liu J, MacLeod JN: Analysis Of Equine Structural Gene Annotation By RNA Sequencing. Plant \& Animal Genomes XVII Conference: 2009; San Diego, CA 2009.

55. Oshlack A, Wakefield MJ: Transcript length bias in RNA-seq data confounds systems biology. Biol Direct 2009, 4:14.

56. Welle S, Bhatt $K$, Thornton CA: Inventory of high-abundance mRNAs in skeletal muscle of normal men. Genome Res 1999, 9(5):506-513.

57. Payne RM, Strauss AW: Expression of the mitochondrial creatine kinase genes. Mol Cell Biochem 1994, 133-134:235-243.

58. Ventura-Clapier R, Kuznetsov A, Veksler V, Boehm E, Anflous K: Functional coupling of creatine kinases in muscles: species and tissue specificity. Mol Cell Biochem 1998, 184(1-2):231-247.

59. Gellerich FN, Khuchua ZA, Kuznetsov AV: Influence of the mitochondrial outer membrane and the binding of creatine kinase to the mitochondrial inner membrane on the compartmentation of adenine nucleotides in the intermembrane space of rat heart mitochondria. Biochim Biophys Acta 1993, 1140(3):327-334.

60. Wallimann T, Wyss M, Brdiczka D, Nicolay K, Eppenberger HM: Intracellular compartmentation, structure and function of creatine kinase isoenzymes in tissues with high and fluctuating energy demands: the 'phosphocreatine circuit' for cellular energy homeostasis. Biochem J 1992, 281(Pt 1):21-40.

61. Gu J, Katz LM, MacHugh DE, McGivney BA, Park SD, Hill EW: Sequence variants in equine CKM (creatine kinase, muscle) and COX4I2 (cytochrome c oxidase, subunit 4, isoform 2) genes are associated with elite racing performance in Thoroughbred horses. International Conference on Equine Exercise Physiology: 2010; Cape Town, South Africa 2010

62. Gu J, Orr N, Park SD, Katz LM, Sulimova G, MacHugh DE, Hill EW: A genome scan for positive selection in thoroughbred horses. PLoS One 2009, 4(6):e5767.

63. MacLeod JN: Analysis Of Equine Gene Expression By RNA Sequencing. Plant \& Animal Genome Conference XVIII: 2009; San Diego, CA, USA 2009.

64. Rivero IL, Galisteo AM, Aguera E, Miro F: Skeletal muscle histochemistry in male and female Andalusian and Arabian horses of different ages. Res Vet Sci 1993, 54(2):160-169.

65. Sekoguchi E, Sato N, Yasui A, Fukada S, Nimura Y, Aburatani H, Ikeda K, Matsuura A: A novel mitochondrial carnitine-acylcarnitine translocase induced by partial hepatectomy and fasting. J Biol Chem 2003, 278(40):38796-38802.

66. Orii KO, Aoyama T, Souri M, Orii KE, Kondo N, Orii T, Hashimoto T: Genomic DNA organization of human mitochondrial very-long-chain acyl-CoA dehydrogenase and mutation analysis. Biochem Biophys Res Commun 1995, 217(3):987-992

67. Matthews CE, Ockene IS, Freedson PS, Rosal MC, Merriam PA, Hebert JR: Moderate to vigorous physical activity and risk of upper-respiratory tract infection. Med Sci Sports Exerc 2002, 34(8):1242-1248.

68. Gleeson M: Mucosal immune responses and risk of respiratory illness in elite athletes. Exerc/mmunol Rev 2000, 6:5-42.
69. Bouwman FG, van Ginneken MM, Noben JP, Royackers E, de GraafRoelfsema E, Wijnberg ID, van der Kolk JH, Mariman EC, van Breda E: Differential expression of equine muscle biopsy proteins during normal training and intensified training in young standardbred horses using proteomics technology. Comp Biochem Physiol Part D Genomics Proteomics 5(1):55-64.

70. Stefansson S, Yepes M, Gorlatova N, Day DE, Moore EG, Zabaleta A, McMahon GA, Lawrence DA: Mutants of plasminogen activator inhibitor-1 designed to inhibit neutrophil elastase and cathepsin $\mathrm{G}$ are more effective in vivo than their endogenous inhibitors. $J$ Biol Chem 2004, 279(29):29981-29987.

71. Dufaux B, Order U: Plasma elastase-alpha 1-antitrypsin, neopterin, tumor necrosis factor, and soluble interleukin-2 receptor after prolonged exercise. Int J Sports Med 1989, 10(6):434-438.

72. Pyne DB: Regulation of neutrophil function during exercise. Sports Med 1994, 17(4):245-258

73. Shephard RJ, Shek PN: Immune responses to inflammation and trauma: a physical training model. Can J Physiol Pharmacol 1998, 76(5):469-472.

74. Moldoveanu Al, Shephard RJ, Shek PN: The cytokine response to physical activity and training. Sports Med 2001, 31(2):115-144

75. Niess AM, Dickhuth HH, Northoff H, Fehrenbach E: Free radicals and oxidative stress in exercise--immunological aspects. Exerc Immunol Rev 1999, 5:22-56

76. Dousset E, Avela J, Ishikawa M, Kallio J, Kuitunen S, Kyrolainen H, Linnamo $\checkmark$, Komi PV: Bimodal recovery pattern in human skeletal muscle induced by exhaustive stretch-shortening cycle exercise. Med Sci Sports Exerc 2007, 39(3):453-460.

77. Phillips SM, Tipton KD, Ferrando AA, Wolfe RR: Resistance training reduces the acute exercise-induced increase in muscle protein turnover. Am J Physiol 1999, 276(1 Pt 1):E118-124.

78. Tipton KD, Ferrando AA, Phillips SM, Doyle D Jr, Wolfe RR: Postexercise net protein synthesis in human muscle from orally administered amino acids. Am J Physiol 1999, 276(4 Pt 1):E628-634.

79. Reppert SM, Weaver DR: Coordination of circadian timing in mammals. Nature 2002, 418(6901):935-941.

80. Oishi K, Sakamoto K, Okada T, Nagase T, Ishida N: Antiphase circadian expression between BMAL1 and period homologue MRNA in the suprachiasmatic nucleus and peripheral tissues of rats. Biochem Biophys Res Commun 1998, 253(2):199-203.

81. Atkinson G, Edwards B, Reilly T, Waterhouse J: Exercise as a synchroniser of human circadian rhythms: an update and discussion of the methodological problems. Eur J App/ Physiol 2007, 99(4):331-341.

82. Stokkan KA, Yamazaki S, Tei H, Sakaki Y, Menaker M: Entrainment of the circadian clock in the liver by feeding. Science 2001, 291(5503):490-493.

83. Edgar DM, Dement WC: Regularly scheduled voluntary exercise synchronizes the mouse circadian clock. Am J Physiol 1991, 261(4 Pt 2):R928-933

84. Piccione G, Grasso F, Fazio F, Giudice E: The effect of physical exercise on the daily rhythm of platelet aggregation and body temperature in horses. Vet J 2008, 176(2):216-220.

85. Murphy BA, Vick MM, Sessions DR, Cook RF, Fitzgerald BP: Evidence of an oscillating peripheral clock in an equine fibroblast cell line and adipose tissue but not in peripheral blood. Journal of comparative physiology 2006, 192(7):743-751.

86. Souissi N, Gauthier A, Sesboue B, Larue J, Davenne D: Effects of regular training at the same time of day on diurnal fluctuations in muscular performance. J Sports Sci 2002, 20(11):929-937.

87. Reilly T, Atkinson G, Edwards B, Waterhouse J, Farrelly K, Fairhurst E: Diurnal variation in temperature, mental and physical performance, and tasks specifically related to football (soccer). Chronobiol Int 2007, 24(3):507-519

88. Brisswalter J, Bieuzen F, Giacomoni M, Tricot V, Falgairette G: Morning-toevening differences in oxygen uptake kinetics in short-duration cycling exercise. Chronobiol Int 2007, 24(3):495-506.

89. Morris C, Atkinson G, Drust B, Marrin K, Gregson W: Human core temperature responses during exercise and subsequent recovery: an important interaction between diurnal variation and measurement site. Chronobiol Int 2009, 26(3):560-575.

90. Suter E, Hoppeler H, Claassen H, Billeter R, Aebi U, Horber F, Jaeger P, Marti B: Ultrastructural modification of human skeletal muscle tissue with 6month moderate-intensity exercise training. Int J Sports Med 1995, 16(3):160-166. 
91. Desplanches D, Hoppeler H, Linossier MT, Denis C, Claassen H, Dormois D, Lacour JR, Geyssant A: Effects of training in normoxia and normobaric hypoxia on human muscle ultrastructure. Pflugers Arch 1993, 425(34):263-267.

92. Howald H, Hoppeler H, Claassen H, Mathieu O, Straub R: Influences of endurance training on the ultrastructural composition of the different muscle fiber types in humans. Pflugers Arch 1985, 403(4):369-376.

93. Hoppeler H, Howald H, Conley K, Lindstedt SL, Claassen H, Vock P, Weibel ER: Endurance training in humans: aerobic capacity and structure of skeletal muscle. J Appl Physiol 1985, 59(2):320-327.

94. MacArthur DG, North KN: ACTN3: A genetic influence on muscle function and athletic performance. Exerc Sport Sci Rev 2007, 35(1):30-34.

95. Hewitt JE: Abnormal glycosylation of dystroglycan in human genetic disease. Biochim Biophys Acta 2009, 1792(9):853-861.

96. Cohn RD: Dystroglycan: important player in skeletal muscle and beyond. Neuromuscul Disord 2005, 15(3):207-217.

97. Chin ER: Role of $\mathrm{Ca} 2+/$ calmodulin-dependent kinases in skeletal muscle plasticity. J App/ Physio/ 2005, 99(2):414-423.

98. Michel RN, Chin ER, Chakkalakal JV, Eibl JK, Jasmin BJ: Ca2+/calmodulinbased signalling in the regulation of the muscle fibre phenotype and its therapeutic potential via modulation of utrophin $A$ and myostatin expression. Appl Physio/ Nutr Metab 2007, 32(5):921-929.

99. Tentori L, Graziani G: Doping with growth hormone/IGF-1, anabolic steroids or erythropoietin: is there a cancer risk? Pharmacol Res 2007, 55(5):359-369.

100. Saugy M, Robinson N, Saudan C, Baume N, Avois L, Mangin P: Human growth hormone doping in sport. Br J Sports Med 2006:135-39.

101. Firth SM, Baxter RC: Cellular actions of the insulin-like growth factor binding proteins. Endocr Rev 2002, 23(6):824-854.

102. Ren $H$, Yin P, Duan C: IGFBP-5 regulates muscle cell differentiation by binding to IGF-II and switching on the IGF-II auto-regulation loop. J Cell Biol 2008, 182(5):979-991.

103. Kuemmerle JF, Zhou H: Insulin-like growth factor-binding protein-5 (IGFBP-5) stimulates growth and IGF-I secretion in human intestinal smooth muscle by Ras-dependent activation of p38 MAP kinase and Erk1/2 pathways. J Biol Chem 2002, 277(23):20563-20571.

104. McPherron AC, Lawler AM, Lee SJ: Regulation of skeletal muscle mass in mice by a new TGF-beta superfamily member. Nature 1997, 387(6628):83-90.

105. Roth SM, Martel GF, Ferrell RE, Metter EJ, Hurley BF, Rogers MA: Myostatin gene expression is reduced in humans with heavy-resistance strength training: a brief communication. Exp Biol Med (Maywood) 2003, 228(6):706-709.

106. Kim JS, Cross JM, Bamman MM: Impact of resistance loading on myostatin expression and cell cycle regulation in young and older men and women. Am J Physiol Endocrinol Metab 2005, 288(6):E1110-1119.

107. Catipovic B: Myostatin mutation associated with gross muscle hypertrophy in a child. N Engl J Med 2004, 351(10):1030-1031. author reply 1030-1031

108. Grobet L, Martin LJ, Poncelet D, Pirottin D, Brouwers B, Riquet J, Schoeberlein A, Dunner S, Menissier F, Massabanda J, et al:: A deletion in the bovine myostatin gene causes the double-muscled phenotype in cattle. Nat Genet 1997, 17(1):71-74.

109. MCPherron AC, Lee SJ: Double muscling in cattle due to mutations in the myostatin gene. Proc Natl Acad Sci USA 1997, 94(23):12457-12461.

110. Mosher DS, Quignon P, Bustamante CD, Sutter NB, Mellersh CS, Parker HG, Ostrander EA: A mutation in the myostatin gene increases muscle mass and enhances racing performance in heterozygote dogs. PLOS Genet 2007, 3(5):e79..

111. Schuelke M, Wagner KR, Stolz LE, Hubner C, Riebel T, Komen W, Braun T, Tobin JF, Lee SJ: Myostatin mutation associated with gross muscle hypertrophy in a child. N Engl J Med 2004, 350(26):2682-2688.

112. Hill EW, Gu J, Eivers SS, Fonseca RG, McGivney BA, Govindarajan P, Orr N, Katz LM, Machugh DE: Correction: A Sequence Polymorphism in MSTN Predicts Sprinting Ability and Racing Stamina in Thoroughbred Horses. PLOS ONE 2010, 5:. 2010/02/04 edn

113. Lindholm A, Piehl K: Fibre composition, enzyme activity and concentrations of metabolites and electrolytes in muscles of standardbred horses. Acta Vet Scand 1974, 15(3):287-309.

114. Dingboom EG, Dijkstra G, Enzerink E, van Oudheusden HC, Weijs WA: Postnatal muscle fibre composition of the gluteus medius muscle of
Dutch Warmblood foals; maturation and the influence of exercise. Equine Vet J Supp/ 1999:95-100.

115. Hubbard TJ, Aken BL, Ayling S, Ballester B, Beal K, Bragin E, Brent S, Chen Y, Clapham P, Clarke L, et al.: Ensembl 2009. Nucleic Acids Res 2009:D690-697.

116. Karolchik D, Kuhn RM, Baertsch R, Barber GP, Clawson H, Diekhans M, Giardine B, Harte RA, Hinrichs AS, Hsu F, et al.: The UCSC Genome Browser Database: 2008 update. Nucleic Acids Res 2008:D773-779.

117. Robinson MD, Smyth GK: Moderated statistical tests for assessing differences in tag abundance. Bioinformatics 2007, 23(21):2881-2887.

118. Dennis G Jr, Sherman BT, Hosack DA, Yang J, Gao W, Lane HC, Lempicki RA: DAVID: Database for Annotation, Visualization, and Integrated Discovery. Genome Biol 2003, 4(5):P3.

119. Huang da W, Sherman BT, Lempicki RA: Systematic and integrative analysis of large gene lists using DAVID bioinformatics resources. Nat Protoc 2009, 4(1):44-57.

120. Hosack DA, Dennis G Jr, Sherman BT, Lane HC, Lempicki RA: Identifying biological themes within lists of genes with EASE. Genome Biol 2003, 4(10):R70

121. Al-Shahrour F, Arbiza L, Dopazo H, Huerta-Cepas J, Minguez P, Montaner $D$, Dopazo J: From genes to functional classes in the study of biological systems. BMC Bioinformatics 2007, 8:114.

122. Al-Shahrour F, Minguez P, Tarraga J, Montaner D, Alloza E, Vaquerizas JM Conde L, Blaschke C, Vera J, Dopazo J: BABELOMICS: a systems biology perspective in the functional annotation of genome-scale experiments. Nucleic Acids Res 2006:W472-476

123. Benjamini Y, Hochberg Y: Controlling the false discovery rate - a practical and powerful approach to multiple testing. Journal of the Royal Statistical Society Series B-Methodological 1995, 57(1):289-300.

124. Cappelli K, Felicetti M, Capomaccio S, Spinsanti G, Silvestrelli M, Supplizi $\mathrm{AV}$ : Exercise induced stress in horses: selection of the most stable reference genes for quantitative RT-PCR normalization. BMC Mol Biol 2008, 9:49.

doi: $10.1186 / 1471-2164-11-398$

Cite this article as: McGivney et al., Characterization of the equine skeletal muscle transcriptome identifies novel functional responses to exercise training BMC Genomics 2010, 11:398

\section{Submit your next manuscript to BioMed Central and take full advantage of:}

- Convenient online submission

- Thorough peer review

- No space constraints or color figure charges

- Immediate publication on acceptance

- Inclusion in PubMed, CAS, Scopus and Google Scholar

- Research which is freely available for redistribution
C) BioMed Central 